\title{
Experimental Verification of a Global Exponentially Stable Nonlinear Wave Encounter Frequency Estimator
}

\author{
Belleter, Dennis J.W.; Galeazzi, Roberto; Fossen, Thor Inge
}

Published in:

Ocean Engineering

Link to article, DOI:

10.1016/j.oceaneng.2014.12.030

Publication date:

2015

Document Version

Early version, also known as pre-print

Link back to DTU Orbit

Citation (APA):

Belleter, D. J.W., Galeazzi, R., \& Fossen, T. I. (2015). Experimental Verification of a Global Exponentially Stable Nonlinear Wave Encounter Frequency Estimator. Ocean Engineering, 97, 48-56.

https://doi.org/10.1016/j.oceaneng.2014.12.030

\section{General rights}

Copyright and moral rights for the publications made accessible in the public portal are retained by the authors and/or other copyright owners and it is a condition of accessing publications that users recognise and abide by the legal requirements associated with these rights.

- Users may download and print one copy of any publication from the public portal for the purpose of private study or research.

- You may not further distribute the material or use it for any profit-making activity or commercial gain

- You may freely distribute the URL identifying the publication in the public portal 


\title{
Experimental Verification of a Global Exponential Stable Nonlinear Wave Encounter Frequency Estimator
}

\author{
Dennis J.W. Belleter ${ }^{\mathrm{a}}$, Roberto Galeazzi ${ }^{\mathrm{b}}$, Thor I. Fossen ${ }^{\mathrm{a}}$ \\ ${ }^{a}$ Centre for Autonomous Marine Operations and Systems (AMOS), Department of \\ Engineering Cybernetics, Norwegian University of Science and Technology, \\ 7491 Trondheim, Norway \\ ${ }^{b}$ Department of Electrical Engineering, Technical University of Denmark, \\ Kgs. Lyngby, Denmark
}

\begin{abstract}
This paper presents a global exponential stability (GES) proof for a signalbased nonlinear wave encounter frequency estimator. The estimator under consideration is a second-order nonlinear observer designed to estimate the frequency of a sinusoid with unknown frequency, amplitude and phase. The GES proof extends previous results that only guarantee global $\mathcal{K}$-exponential stability. Typical applications are control and decision-support systems for marine craft, where it is important to know the sea state and wave frequency. The theoretical results are verified experimentally by analyzing data from towing tank experiments using a container ship scale model. The estimates for both regular and irregular waves confirm the results. Finally, the estimator is applied to full-scale data gathered from a container ship operating in the Atlantic Ocean during a storm. Again the theoretical results are confirmed.
\end{abstract}

Keywords: Nonlinear observer, marine systems, sea-state estimation

\section{Introduction}

Estimation of the wave encounter frequency is an important part of seastate prediction, which is of great importance in many marine operations as well as control systems design. Sea-state estimation also provides information

Email addresses: dennis.belleter@itk.ntnu.no (Dennis J.W. Belleter), rg@elektro.dtu.dk (Roberto Galeazzi), fossen@ieee.org (Thor I. Fossen) 
to increase both the safety of operations at sea and the performance of control systems for ships affected by waves.

To increase the safety of operations, knowledge of the wave encounter frequency can be used for prediction of extreme waves, parametric roll resonance and in-service monitoring. Knowledge of the sea-state, and in particular the encounter frequency, is also important to increase the performance of marine control systems. For ship autopilot and dynamic positioning (DP) systems knowledge of the encounter frequency allows for better tuning of the low-pass and notch filters used in wave filtering (Fossen, 2011, Ch. 11). On-line adjustment of controller and observer gains also require knowledge of the wave encounter frequency (Fossen and Strand, 1999). This allows for automatic gain scheduling of autopilots and DP systems.

\subsection{Sea-state estimation}

In the literature several techniques for estimation of the wave encounter frequency or wave spectra have been presented. The classical method is to obtain the wave spectrum from Fast Fourier Transform (FFT) frequency spectral analysis (Enshaei and Birmingham, 2012). Unfortunately, creating a FFT frequency spectrum takes time and consequently it results in back-dated information when estimating the time-varying wave encounter frequency. This is due to the moving window necessary for applying the FFT frequency spectral analysis. Hence, it is impossible to estimate a timevarying wave encounter frequency without lag.

More advanced spectral estimation techniques allow estimation of directional wave spectra (Nielsen, 2006). This can be done by parametric or non-parametric modeling. The parametric modeling approach typically assumes that the wave spectrum is parametrized such that it can be estimated using least-squares parameter matching of a bimodal spectrum for stationary vessels (Tannuri et al., 2003) and moving vessels (Nielsen, 2006). The non-parametric modeling or Bayesian approach uses stochastic processes to match the frequencies for stationary vessels (Iseki and Ohtsu, 2000) and moving vessels (Nielsen, 2006). These techniques have the same disadvantages with respect to acquisition times as the FFT frequency spectral analysis. However, besides the frequency of the waves, they also supply directional information.

Another approach to wave encounter frequency estimation is to estimate the peak frequency instead of the entire wave spectrum. This is a valid approach for application of sea-state estimation when designing control systems, 
since the peak frequency of the spectrum is used for wave filtering (Fossen, 2011, Ch. 11). Approaches using Kalman filters can be found in Belleter et al. (2012) and Hassani et al. (2013). However, these approaches require a dynamic model of the vessel. A signal-based approach was developed in Belleter et al. (2013), where the measured roll or pitch angle is used to estimate the wave frequency. This approach is taken in this paper and experimental verification is included to justify the results.

\subsection{Frequency estimation}

Frequency estimation of oscillating signals is a well studied problem in the signal processing literature. A discrete-time algorithm for a multifrequency signal based on an adaptive notch filtering was first proposed by Regalia (1991). A continuous-time version of this algorithm was presented in Bodson and Douglas (1997), while Hsu et al. (1999) have derived a globally convergent continuous-time frequency estimator for a single frequency signal.

An adaptive technique based on the persistency of excitation (PE) of oscillating signals was proposed in Marino and Tomei (2002), and extended by Xia (2002) and Hou (2012). Two discrete-time algorithms based on PE can also be found in Stotsky (2012).

The approach taken by the authors is based on the internal model principle for identification of a single frequency. This was first introduced in Nikiforov (1997) and further extended by Aranovskiy et al. (2007), Bobtsov (2008), and Aranovskiy and Bobtsov (2012).

\subsection{Main contribution}

The main result of the paper is a nonlinear signal-based wave encounter frequency estimator, which effectively estimates the ship wave encounter frequency from heave, pitch or roll motion measurements. The wave encounter frequency estimator under consideration is designed to estimate the frequency of a sinusoid with unknown frequency, amplitude and phase by modifying the algorithm of Aranovskiy et al. (2007) to include an adaptive gain-switching mechanism. The GES proof extends previous results (Belleter et al., 2013) that only guarantee uniform global asymptotic stability (UGAS) and uniform local exponential stability (ULES). This is also referred to as global $\mathcal{K}$-exponential stability (Sørdalen and Egeland, 1995).

The main motivation for introducing a gain-switching mechanism is that it is important to improve the convergence of the estimator in situations with little excitation (e.g. small roll and pitch angles) and vice versa. Typical 
applications are marine craft control and decision-support systems where it is important to know the sea state and wave frequency. The wave encounter frequency estimator is experimentally verified through towing tank tests in both regular and irregular waves. The estimator is also verified for 9-hours of data gathered onboard the container vessel Clara Maersk during a storm across the North Atlantic Ocean.

The wave estimator is implemented in real-time and consequently it is much faster than the real-time requirement of the ship autopilot and DP control systems, which typically samples data at $1-10 \mathrm{~Hz}$. FFT is an off-line algorithm, which use batches of data (moving window). The computational footprint is higher and significantly affected by the acquisition time and numerical processing of the data.

\subsection{Organization of the paper}

The paper is organized as follows: In Section 2 the wave encounter frequency estimation problem is introduced and the Aranovskiy fixed-gain frequency estimator is reviewed. Section 3 presents the switching-gain frequency estimator and GES of the equilibrium point of the estimation error dynamics is proven. Section 4 contains experimental verification using towing tank experiments and full-scale data of a container ship.

\section{Estimation of the Wave Spectrum Encounter Frequency}

Characterization of the sea state for marine operations is generally done in terms of a limited number of fundamental parameters, which are used to calculate approximations of the wave spectrum. Those parameters are the significant wave height $H_{s}$, the wave modal frequency (peak frequency) $\omega_{0}$, and the wave encounter angle $\beta_{e}$ that is the relative angle between the vessel heading and the the main direction of the wave train. Knowledge of those parameters may reveal to be of extreme importance in order to schedule and perform activities at sea in a safe, reliable and cost effective manner.

For vessels in transit at forward speed $U>0$ the experienced wave excitation does not occur at the modal frequency $\omega_{0}$ because of the Doppler shift. The frequency observed from the vessel in motion is given by:

$$
\omega_{e}\left(\omega_{0}, U, \beta_{e}\right)=\left|\omega_{0}-\frac{\omega_{0}^{2}}{g} U \cos \left(\beta_{e}\right)\right|
$$


which is known as the wave encounter frequency. Awareness about $\omega_{e}$ would allow performance enhancement of ship control systems. For instance autopilots and DP systems use wave filters, which are tuned to suppress oscillations at the encounter frequency, in order to reduce the workload of the steering and propulsion systems.

Although waves are usually described as narrow-band stochastic processes, the associated spectrum is certainly richer in frequency content than a single sinusoid. Nevertheless spectral analysis of wave-induced vessel motions usually displays a dominant frequency associated with the peak of the spectrum. During the transient the natural frequencies of the different modes can be observed in the spectrum giving rise to multiple peaks. However, If the waves are large enough the ship will oscillate at $\omega_{e}$ in all 6 degrees-offreedom in steady state. For multi-peaked wave spectra with a dominant peak the proposed method will provide an estimate close to the frequency of the highest peak. Analytically the problem can be formulated as:

\section{Problem definition (Wave encounter frequency estimation)}

Given the signal in the form:

$$
y(t)=A(t) \sin \left(\omega_{e} t+\epsilon\right)
$$

with $A(t)$ the unknown amplitude, $\omega_{e}$ the unknown frequency and $\epsilon$ the unknown phase, reconstruct on-line the frequency $\omega_{e}$ based solely on noisy measurements of $y(t)$.

\subsection{The Aranovskiy fixed-gain frequency estimator}

Before presenting the main contribution of the paper (Theorem 1), we first review the signal-based frequency estimator proposed by Aranovskiy et al. (2007), which is instrumental in our design.

The sinusoidal signal (2) can be represented by the differential equation:

$$
\ddot{y}=\varphi y
$$

where $\varphi:=-\omega_{e}^{2}$ is treated as an unknown parameter. The frequency $\omega_{e}$ of the signal (2) can be estimated using an auxiliary filter (Aranovskiy and Bobtsov, 2012):

$$
\begin{aligned}
& \dot{\zeta}_{1}=\zeta_{2} \\
& \dot{\zeta}_{2}=-2 \omega_{f} \zeta_{2}-\omega_{f}^{2} \zeta_{1}+\omega_{f}^{2} y
\end{aligned}
$$


where the filter cut-off frequency must be chosen such that $0<\omega_{e}<\omega_{f}$. The transfer function corresponding to (4)-(5) is found by Laplace transformation:

$$
\zeta_{1}(s)=\frac{\omega_{f}^{2}}{\left(s+\omega_{f}\right)^{2}} y(s)
$$

From (3) it follows that $s^{2} y(s)=\varphi y(s)$ and

$$
\begin{aligned}
y(s) & =\frac{\varphi+2 \omega_{f} s+\omega_{f}^{2}}{\left(s+\omega_{f}\right)^{2}} y(s) \\
& =\frac{2 \omega_{f} s+\omega_{f}^{2}+\varphi}{\omega_{f}^{2}} \zeta_{1}(s)
\end{aligned}
$$

Transforming this expression to the time domain gives:

$$
y=\frac{1}{\omega_{f}^{2}}\left(2 \omega_{f} \zeta_{2}+\omega_{f}^{2} \zeta_{1}+\varphi \zeta_{1}\right)
$$

The Aranovskiy et al. (2007) parameter update law for $\varphi$ uses the computed measurement:

$$
y^{\prime}:=\dot{\zeta}_{2}=-2 \omega_{f} \zeta_{2}-\omega_{f}^{2} \zeta_{1}+\omega_{f}^{2} y=\varphi \zeta_{1}
$$

Let $\hat{\varphi}$ denote the parameter estimate and define:

$$
\hat{y}^{\prime}:=\hat{\varphi} \zeta_{1}
$$

The parameter update law is chosen as:

$$
\dot{\hat{\varphi}}=k_{0} \zeta_{1}\left(y^{\prime}-\hat{y}^{\prime}\right)
$$

where $k_{0}>0$ is the constant observer gain. Consequently, the resulting frequency estimator becomes:

$$
\begin{aligned}
\dot{\zeta}_{1} & =\zeta_{2} \\
\dot{\zeta}_{2} & =-2 \omega_{f} \zeta_{2}-\omega_{f}^{2} \zeta_{1}+\omega_{f}^{2} y \\
\dot{\hat{\varphi}} & =k_{0} \zeta_{1}\left(\dot{\zeta}_{2}-\hat{\varphi} \zeta_{1}\right)
\end{aligned}
$$

The differential equation for the parameter estimation error $\tilde{\varphi}=\varphi-\hat{\varphi}$ where $\varphi$ is assumed to be constant becomes:

$$
\dot{\tilde{\varphi}}=-k_{0} \zeta_{1}^{2} \tilde{\varphi}
$$


The wave component (2) has a positive amplitude $0<A_{\min } \leq A$ for $\forall t \geq 0$ and frequency $\omega_{e}>0$. For frequencies $\omega_{e}<\omega_{f}$, the time-domain solution of (6) for a sinusoidal input (2) is $\zeta_{1}=A \sin \left(\omega_{e} t+\epsilon_{1}\right)$ where $\epsilon_{1}$ is the phase. The signal $\zeta_{1}$ is persistently exciting (PE) since there exist a positive $\mu$ and $T$ such that

$$
\mu \leq \int_{t}^{t+T} \zeta_{1}^{2}(\tau) \mathrm{d} \tau, \quad \forall t \geq 0
$$

The PE-condition (16) is used to prove that the the equilibrium point of the estimation error dynamics (15) is GES for constant adaptation gain $k_{0}>0$ and $A>0$. This result will be generalized to time-varying adaptation gain and wave amplitude in Section 3.

The solutions of (15) satisfy:

$$
\begin{aligned}
\|\tilde{\varphi}\| & =\left\|\tilde{\varphi}\left(t_{0}\right)\right\| e^{-k_{0} \int_{t_{0}}^{t} \zeta_{1}^{2}(\tau) \mathrm{d} \tau} \\
& =\left\|\tilde{\varphi}\left(t_{0}\right)\right\| e^{-k_{0} \int_{t_{0}}^{t} A^{2} \sin ^{2}\left(\omega_{e} \tau+\epsilon_{1}\right) \mathrm{d} \tau}
\end{aligned}
$$

with

$$
\begin{aligned}
\int_{t_{0}}^{t} A^{2} \sin ^{2}\left(\omega_{e} \tau+\epsilon_{1}\right) \mathrm{d} \tau= & \frac{A^{2}}{2}\left(t-t_{0}\right)-\frac{A^{2}}{4 \omega_{e}} \sin \left(2 \omega_{e} t+2 \epsilon_{1}\right) \\
& +\frac{A^{2}}{4 \omega_{e}} \sin \left(2 \omega_{e} t_{0}+2 \epsilon_{1}\right)
\end{aligned}
$$

Substituting (18) in (17) gives:

$$
\begin{aligned}
\|\tilde{\varphi}\| & =\left\|\tilde{\varphi}\left(t_{0}\right)\right\| e^{-\frac{k_{0} A^{2}}{2}\left(t-t_{0}\right)} e^{\frac{k_{0} A^{2}}{4 \omega_{e}}\left[\sin \left(2 \omega_{e} t+2 \epsilon_{1}\right)-\sin \left(2 \omega_{e} t_{0}+2 \epsilon_{1}\right)\right]} \\
& \leq c\left\|\tilde{\varphi}\left(t_{0}\right)\right\| e^{-\lambda\left(t-t_{0}\right)}
\end{aligned}
$$

where $c=e^{k_{0} A^{2} / 2 \omega_{e}}>0$ and $\lambda=k_{0} A^{2} / 2>0$. Hence, by Khalil (2012, Definition 4.5$)$ the equilibrium point $\tilde{\varphi}=0$ of (15) is GES.

Remark 1 (Filter cut-off frequency). The choice of the cut-off frequency $\omega_{f}$ should be made based on desired performance - i.e. convergence rate and steady state error - and noise filtering capabilities. $\omega_{f}$ clearly influences the convergence rate of the estimator as shown in Figure 1, where the frequency of a sinusoidal function oscillating at $0.5 \mathrm{rad} / \mathrm{s}$ is estimated for increasing value of the cut-off frequency. Small cut-off frequencies result in a slow convergence rate. However, higher cut-off frequencies introduce some oscillations 


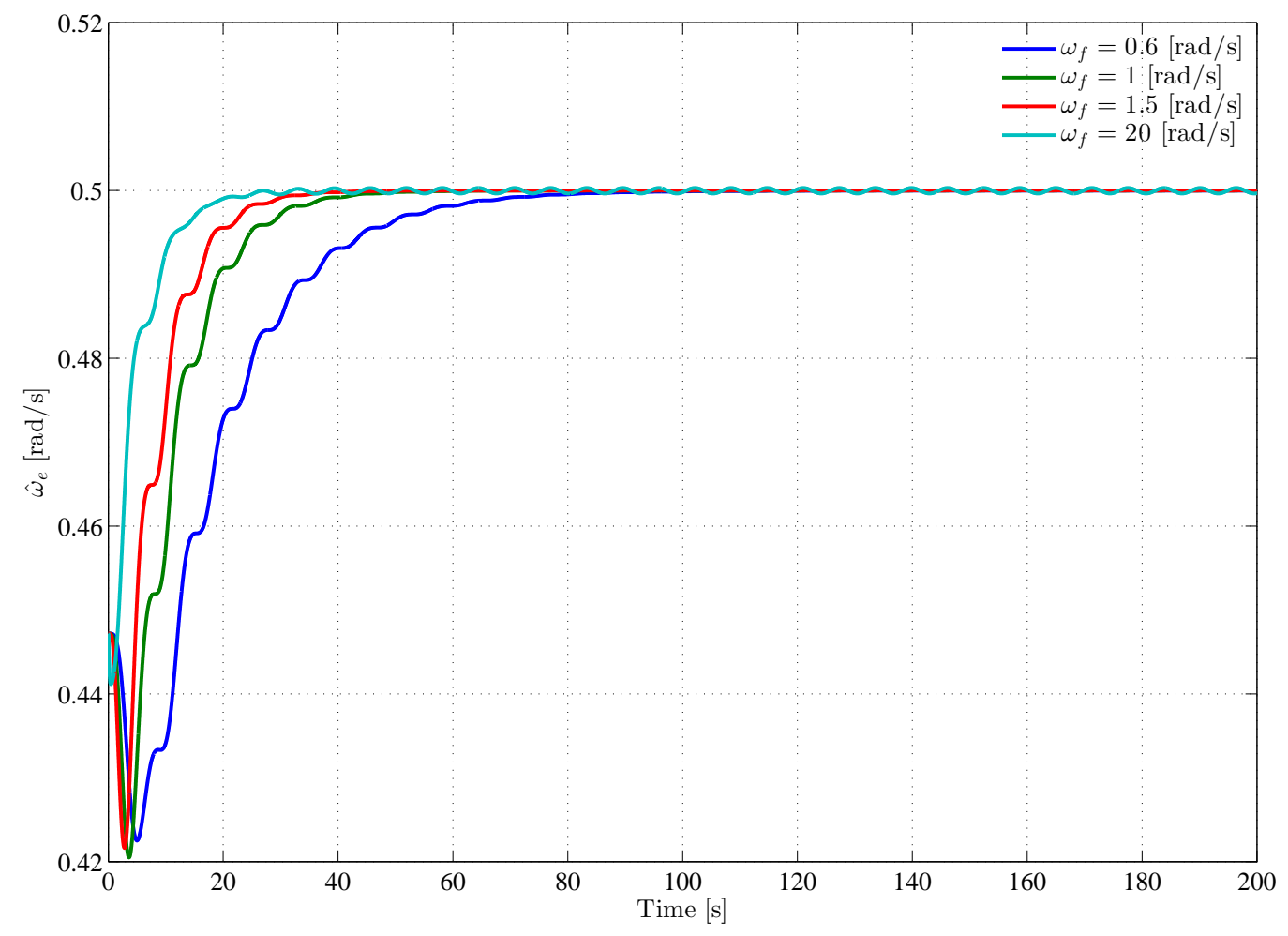

Figure 1: Frequency estimates using different cut-off frequencies $\omega_{f}$.

in steady-state, as visible from the estimate done with $\omega_{f}=20 \mathrm{rad} / \mathrm{s}$. It is interesting to note that moderately increasing the cut-off frequency can significantly increase the convergence rate while the increase in steady-state error is negligible.

\section{Switching-Gain Wave Encounter Frequency Estimator}

The GES stability proof for the Aranovskiy frequency estimator (Section 2.1 ) is based on a constant observer gain $k_{0}>0$. For marine craft the roll and pitch angles may be quite small when operating in low sea states. Consequently, it is advantageous to switch between a high and low gain in the parameter update law depending on the amplitude of the pitch angle. In Belleter et al. (2013) it was shown that the frequency estimator (14) could be modified to include a switching gain $k(A)$, which depends on the amplitude 
$A$ of the measured signal. Moreover,

$$
k(A)= \begin{cases}k_{\text {init }} & \text { if, } t \leq t_{\text {init }} \\ k_{\text {min }} & \text { if, } t>t_{\text {init }} \wedge A>A_{0} \\ k_{\max } & \text { if, } t>t_{\text {init }} \wedge A \leq A_{0}\end{cases}
$$

Here $k_{\text {init }} \geq k_{\min }>0$ is the initial gain used to increase the convergence rate at start up. During normal operation the gain is switched between the positive gains $k_{\min }$ and $k_{\max }$. Moreover, the gain $k(A)$ will switch to the high value if the amplitude $A \leq A_{0}$ and to the low gain when $A>A_{0}$.

To implement the switching mechanism (20) online we need to know the amplitude $A$ of the measured signal $y$. Since we cannot measure $A$ an estimator based on the squared signal of (2) can be used for switching. Moreover,

$$
y^{2}=\frac{A^{2}}{2}\left(1-\cos \left(2 \omega_{e} t+2 \epsilon\right)\right)
$$

The signal (21) can be low-pass filtered to obtain the amplitude $A^{2} / 2$ of the squared signal $y^{2}$. For instance,

$$
\chi=\frac{1}{T s+1} y^{2}
$$

where $T>0$ implies that the estimated amplitude becomes:

$$
\hat{A}=\sqrt{2 \chi}
$$

\subsection{Wave encounter frequency estimator with switching-gain}

The results of Belleter et al. (2013) are in this section extended to be GES for time-varying wave amplitude $A(t)$ and adaptation gain $k_{f}(t)$ by introducing a low-pass filter for the gain $k(\hat{A})$ according to:

$$
T_{f} \dot{k}_{f}+k_{f}=k(\hat{A})
$$

where $T_{f}>0$ is the filter time constant and $k(\hat{A}) \leq \max \left(k_{\max }, k_{\text {init }}\right)$. The parameter update law (14) is modified according to:

$$
\dot{\hat{\varphi}}=k_{f} \zeta_{1}\left(\dot{\zeta}_{2}-\zeta_{1} \hat{\varphi}\right)
$$

and GES is guaranteed by Theorem 1, which is presented below. The effect of low-pass filtering on the gain switching is illustrated in Figure 2. 


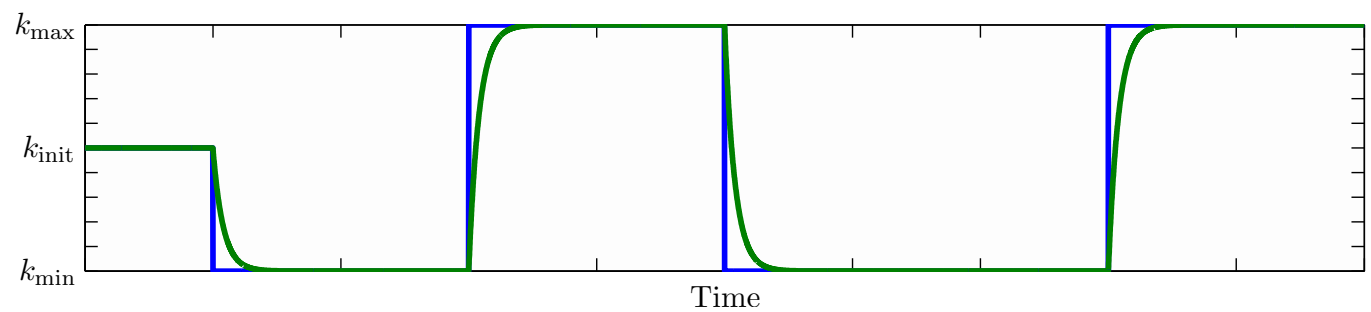

Figure 2: Illustration of low-pass filtered gain changes for step inputs $k(\hat{A})$.

Theorem 1 (GES switching-gain estimator). Let the time-varying wave amplitude satisfy $0<A_{\min } \leq A(t)$ for all $t \geq 0$. Assume that $\varphi=-\omega_{e}^{2}$ is constant and that $k_{f}(t)$ is the solution of (24) for $T_{f}>0$ and step input $k(\hat{A})$ given by $(20)$. Then the equilibrium point $\tilde{\varphi}=0$ of the estimation error dynamics:

$$
\dot{\tilde{\varphi}}=-k_{f} \zeta_{1}^{2} \tilde{\varphi}
$$

is GES.

Proof. From Formulae (20) and (24) it follows that $0<k_{\min } \leq k_{f}(t) \leq k_{\max }$ for all $\hat{A}$. The solutions of (26) satisfy:

$$
\begin{aligned}
\|\tilde{\varphi}\| & =\left\|\tilde{\varphi}\left(t_{0}\right)\right\| e^{-\int_{t_{0}}^{t} k_{f} \zeta_{1}^{2}(\tau) \mathrm{d} \tau} \\
& \leq\left\|\tilde{\varphi}\left(t_{0}\right)\right\| e^{-k_{\min } \int_{t_{0}}^{t} A^{2} \sin ^{2}\left(\omega_{e} \tau+\epsilon_{1}\right) \mathrm{d} \tau} \\
& \leq\left\|\tilde{\varphi}\left(t_{0}\right)\right\| e^{-k_{\min } A_{\min }^{2} \int_{t_{0}}^{t} \sin ^{2}\left(\omega_{e} \tau+\epsilon_{1}\right) \mathrm{d} \tau}
\end{aligned}
$$

Application of (18) to (27) gives:

$$
\begin{aligned}
\|\tilde{\varphi}\| & \leq\left\|\tilde{\varphi}\left(t_{0}\right)\right\| e^{-\frac{k_{\min } A_{\min }^{2}}{2}\left(t-t_{0}\right)} e^{\frac{k_{\min } A_{\min }^{2}}{2 \omega_{e}}} \\
& \leq c\left\|\tilde{\varphi}\left(t_{0}\right)\right\| e^{-\lambda\left(t-t_{0}\right)}
\end{aligned}
$$

where $c=e^{k_{\min } A_{\min }^{2} / 2 \omega_{e}}>0$ and $\lambda=k_{\min } A_{\min }^{2} / 2>0$. Hence, by Khalil (2012, Definition 4.5) the equilibrium point $\tilde{\varphi}=0$ of (26) is GES.

\subsection{Low-pass filtering of the wave encounter frequency estimate}

Since the measured ship motions in general display a non-pure sinusoidal behavior due to the narrow-band spectral characteristic of the wave motions, the estimate $\hat{\varphi}$ provided by (25) will show high-frequency fluctuations as 
a result. Therefore, if the encounter frequency estimator is to be used in applications such as adaptive wave filtering or gain-scheduling control, the high-frequency variations are certainly undesirable since they may introduce chattering in the system. A straightforward solution to this problem is to apply a low-pass filter at the output of the estimator (25), to obtain a running mean of the encounter frequency estimate. Consider the system:

$$
\begin{aligned}
\dot{\hat{\varphi}} & =k_{f} \zeta_{1}\left(\dot{\zeta}_{2}-\zeta_{1} \hat{\varphi}\right) \\
\dot{\varphi}_{f} & =\mathbf{A} \varphi_{f}+\mathbf{b} \hat{\varphi}
\end{aligned}
$$

where $\boldsymbol{\varphi}_{f} \in \mathbb{R}^{n}$ is the vector whose first component $\varphi_{f, 1}$ is the low-pass filtered $\hat{\varphi}$. The Hurwitz design matrix $\mathbf{A}$ and the vector $\mathbf{b}=[0,0,0, \ldots, 1]^{\mathrm{T}}$ define the low-pass filter. By rewriting (29) in terms of the estimation error $\tilde{\varphi}$, and by applying a change of coordinates $\boldsymbol{\xi}=\boldsymbol{\varphi}_{f}+\mathbf{A}^{-1} \mathbf{b} \varphi$ the following cascaded system is obtained:

$$
\begin{array}{ll}
\Sigma_{1}: & \dot{\tilde{\varphi}}=-k_{f} \zeta_{1}^{2} \tilde{\varphi} \\
\Sigma_{2}: & \dot{\boldsymbol{\xi}}=\mathbf{A} \boldsymbol{\xi}+\mathbf{b} \tilde{\varphi}
\end{array}
$$

GES of the cascade $\Sigma_{1}-\Sigma_{2}$ is guaranteed by Corollary 1 .

Corollary 1 (GES cascade). The origin of the cascade $\Sigma_{1}-\Sigma_{2}$ is GES.

Proof. The origin of (31) is GES according to Theorem 1. For $\tilde{\varphi}=0$ the differential equation (32) reduces to $\dot{\boldsymbol{\xi}}=\mathbf{A} \boldsymbol{\xi}$ (nominal system) whose origin is GES since $\mathbf{A}$ is Hurwitz. In addition, the linear growth condition $\|\mathbf{b} \tilde{\varphi}\| \leq|\tilde{\varphi}|$ is satisfied for all $\tilde{\varphi}$. Hence, according to Loria and Panteley (2004, Theorem 2.1, Proposition 2.3) the origin of the cascade $\Sigma_{1}-\Sigma_{2}$ is GES.

\section{Experimental Verification}

The performance of the estimator (20)-(25) in Section 3 is tested on experimental and full-scale data using heave and pitch data. This to demonstrate that the wave frequency estimator performs equally well for both signals. Operationally this will provide flexibility for the operator, which could use measurements provided by either a heave accelerometer or a pitch rate gyro. First, the wave encounter frequency estimator is applied to experimental data gathered through towing tank experiments. Subsequently, the estimator is applied to full-scale sea trial data gathered aboard the container vessel Clara Maersk in an Atlantic passage during a storm. 


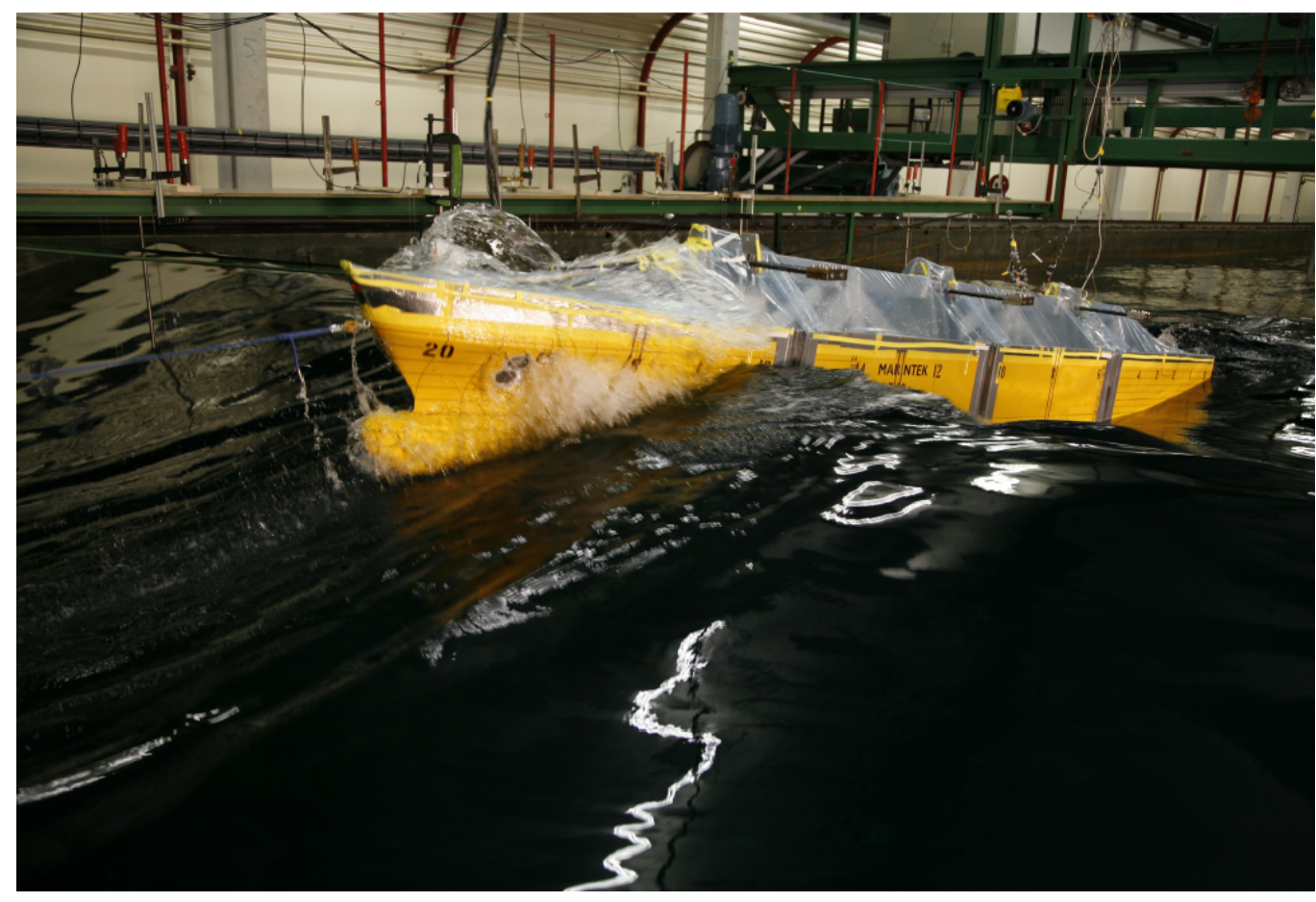

Figure 3: Model ship in the towing tank. Photo courtesy of Dr I. Drummen.

\subsection{Towing tank experiments}

The experimental data considered here was gathered through towing tank tests with a 1:45 scale model of $281 \mathrm{~m}$ long container ship with volume displacement $76000 \mathrm{~m}^{3}$ (see Figure 3). The detailed model and all the hydrodynamic coefficients can be found in Holden et al. (2007).

Table 1: Towing-tank experiment (full scale equivalents)

\begin{tabular}{ccccc}
\hline Exp. & $U[\mathrm{~m} / \mathrm{s}]$ & $\omega_{0}[\mathrm{rad} / \mathrm{s}]$ & $H_{w}[\mathrm{~m}]$ & $\omega_{e}[\mathrm{rad} / \mathrm{s}]$ \\
\hline$R-1173$ & 5.4806 & 0.4425 & 2.5 & 0.5519 \\
$R-1189$ & 7.6675 & 0.4640 & 2.5 & 0.6324 \\
$I-1195$ & 6.0240 & 0.4640 & 9 & 0.5963 \\
\hline
\end{tabular}

The experimental conditions are reported in Table 1. For the regular wave experiments $(R-1173$ and $R-1189) \omega_{0}$ is the wave frequency and $H_{w}$ is the wave amplitude. For the irregular wave experiment $(I-1195) \omega_{0}$ is the 

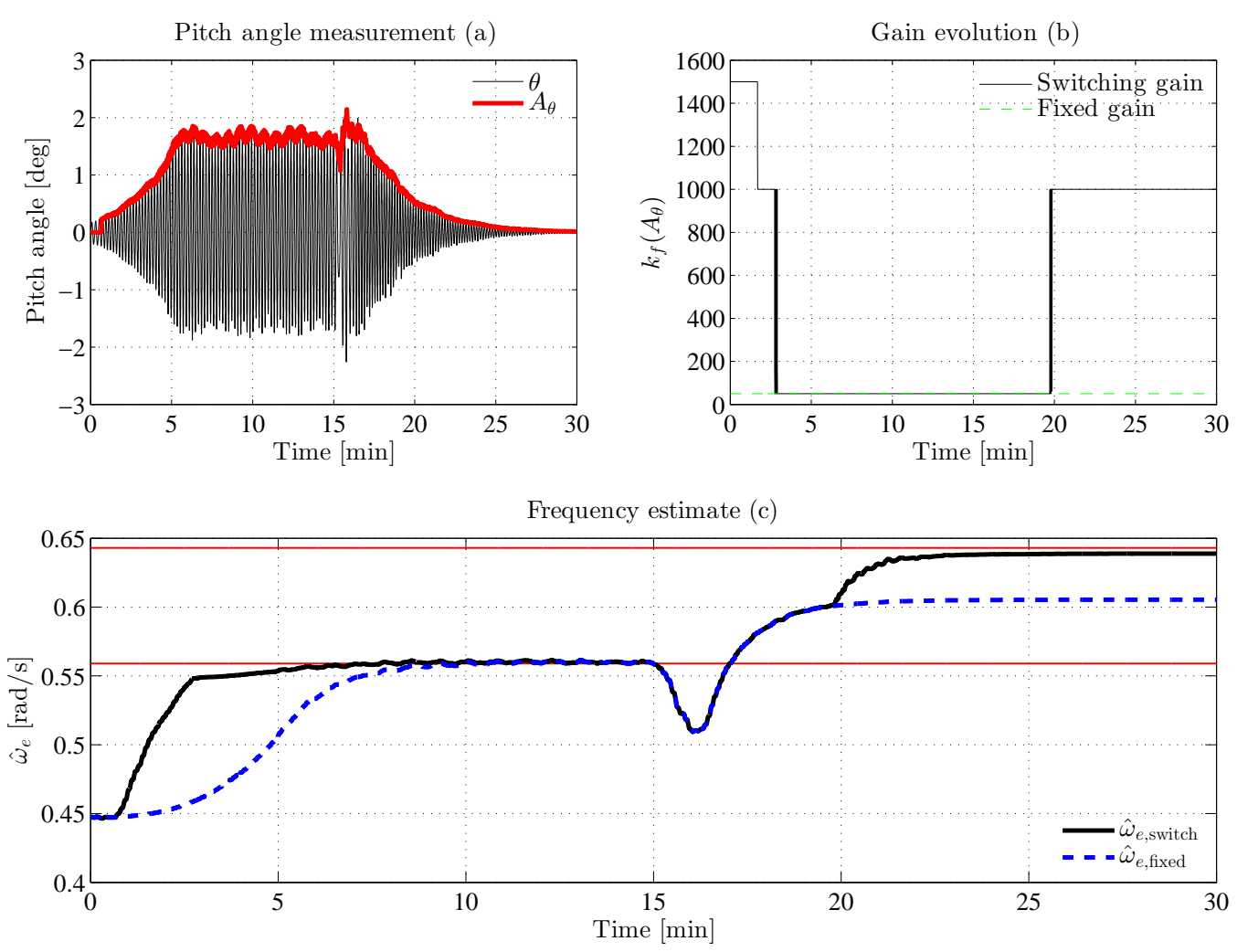

Figure 4: Comparison of the frequency estimator with and without a gain switching mechanism.

peak wave frequency and $H_{w}$ is the significant wave height. $U$ and $\omega_{e}$ are the ship forward speed and the wave encounter frequency, respectively.

\subsubsection{Comparative study of the fixed and variable gain estimators}

The data set R-1173 is used for comparing the performance of the Aranovskiy fixed-gain frequency estimator with the proposed switching-gain solution (Theorem 1). This data set is well suited for this comparison because the amplitude variations of the pitch angle emphasizes the importance of the switching mechanism. The parameters settings for both estimators can be fond in Table 2. Note that the value of $k_{0}$ is chosen equal to $k_{\min }$. This choice guarantees a small steady-sate error during the first 15 minutes of the measurement where the pitch angle shows large amplitudes.

Figure 4 illustrates the results of the comparative study. The use of the very large initialization gain $k_{\text {init }}$ (Figure $4(\mathrm{~b})$ ) boosts the convergence 
Table 2: Parameter settings for the comparative study.

\begin{tabular}{lccc}
\hline Quantity & Symbol & Value & Unit \\
\hline Switching time constant & $T_{f}$ & 0.05 & $\mathrm{~s}$ \\
Filter cut-off frequency & $\omega_{f}$ & 1 & $\mathrm{rad} / \mathrm{s}$ \\
Switching amplitude pitch & $A_{\theta, 0}$ & 0.01 & $\mathrm{rad}$ \\
Initialization time & $t_{\text {init }}$ & 100 & $\mathrm{~s}$ \\
Initialization gain & $k_{\text {init }}$ & 1500 & - \\
Filter low gain & $k_{\text {low }}$ & 50 & - \\
Filter high gain & $k_{\text {high }}$ & 1000 & - \\
Fixed gain & $k_{\text {fixed }}$ & 50 & - \\
\hline
\end{tabular}

rate of the switching-gain estimator, which settles to the true value of the encounter frequency approximately 4 minutes before the fixed-gain estimator (Figure 4(c)). After both filters have converged they have the same gain, and hence the same small steady-state error. When the encounter frequency changes the estimators initially converge to the new value at the same rate. However, when the amplitude gets below the switching threshold $A_{0}$ the switching-gain estimator switches to the much high gain $k_{\max }$, as shown in Figure 4(b). This allows faster convergence towards the correct frequency before the excitation becomes too small. The fixed-gain estimator does not converge to the true value because the gain is to small for limited excitation.

\subsubsection{Performance assessment on towing tank data}

The estimator is tested on pitch and heave measurements gathered during experiments $R-1173$ and $R-1189$, and on a heave measurement gathered during the experiment $I-1195$. The settings for the filter and gain switching parameters are given in Table 3.

At the first the frequency estimator is tested on a measurement of the pitch angle $\theta(t)$, as shown in Figure 5(a). The 30 minutes long time series is obtained by joining the pitch angles measured in experiments $R-1173$ and $R-1189$, and the last 15 minutes of the recording have been amplitude modulated through a decaying exponential function. This behavior has been appositely introduced in order to test the capability of the estimator to track frequency variations in vanishing signals.

The spectral analysis shown in Figure 5(b) clearly shows a frequency shift between the first 15 minutes of the experiments and the second ones in total agreement with the data reported in Table 1 . Moreover, the nature of the excitation used in the regular wave experiments determines a very narrow- 

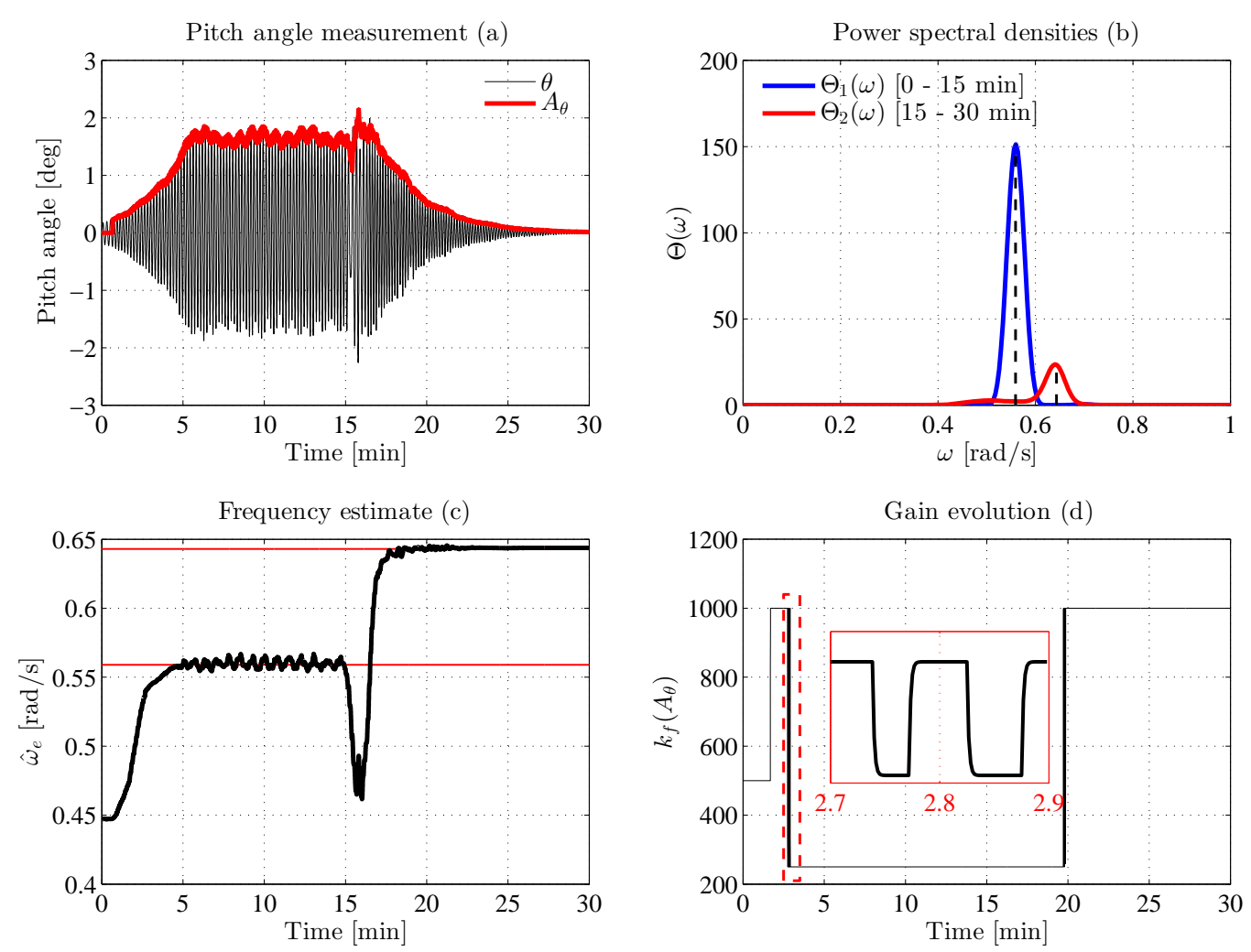

Figure 5: Test I: estimation of wave encounter frequency from exponentially modulated pitch angle measurements collected during regular wave experiments $R-1173$ and $R-1189$. The estimator rapidly converges to and track the peak frequency of the power spectral density $\Theta(\omega)$.

band power spectral density (PSD) denoted $\Theta(\omega)$, and this will ease the estimation process.

The estimate of the wave encounter frequency $\hat{\omega}_{e}$ and the changes in the gain $k_{f}$ can be seen in Figures 5(c)-(d). The two horizontal lines in the frequency estimate plot correspond to the peaks in the PSD's. Figure 5(c) shows that the estimate $\hat{\omega}_{e}$ rapidly converges to the frequency associated to the largest peak of $\Theta_{1}(\omega)$. During the transition to the exponential decaying pitch angle the frequency estimate drops to values in the neighborhood of the small side-lobe of the PSD $\Theta_{2}(\omega)$ and then converges to the frequency value associated with the largest peak of $\Theta_{2}(\omega)$.

Figure 6(a) illustrates the measurement of the heave displacement $z(t)$ recorded during the regular wave experiments. The power spectral density 
Table 3: Switching-gain estimator parameter settings for towing-tank data.

\begin{tabular}{lccc}
\hline Quantity & Symbol & Value & Unit \\
\hline Switching time constant & $T_{f}$ & 0.05 & $\mathrm{~s}$ \\
Filter cut-off frequency & $\omega_{f}$ & 1 & $\mathrm{rad} / \mathrm{s}$ \\
Switching amplitude pitch & $A_{\theta, 0}$ & 0.01 & $\mathrm{rad}$ \\
Switching amplitude heave & $A_{z, 0}$ & 0.6 & $\mathrm{~m}$ \\
Initialization time & $t_{\text {init }}$ & 100 & $\mathrm{~s}$ \\
Initialization gain & $k_{\text {init }}$ & 500 & - \\
Filter low gain & $k_{\min }$ & 250 & - \\
Filter high gain & $k_{\max }$ & 1000 & - \\
\hline
\end{tabular}

$Z(\omega)$ shown in Fig. 6(b) obviously confirms the position of the peak frequencies already identified in the PSD of the pitch angle. The estimate of the wave encounter frequency is not as sharp as seen in relation to the pitch measurement, as shown in Figure 6(c). This reduced precision in estimating the correct value of $\omega_{e}$ may be explained by the presence of side lobs in both PSDs $Z_{1}(\omega)$ and $Z_{2}(\omega)$. If fact for both tranches of the heave measurement the estimate $\hat{\omega}_{e}$ is pulled towards slightly higher frequency values clearly addressing the influence of the side lobes in the estimation process. Last, Figure $6(\mathrm{~d})$ shows that the switching-gain strategy is very active due to the large variability of the heave amplitude $A_{z}$.

At last the switching-gain frequency estimator is tested on measurements of the heave displacement recorded during the irregular waves experiment $I-$ 1195, as shown in Figure 7(a). The large significant wave height together with the irregular pattern of the wave train exciting the vessel determine repeated large and asymmetric variations of the heave displacement. This behavior largely differs from the sinusoidal one and challenges the frequency estimator, as shown in Figure $7(\mathrm{c})$. The estimate $\hat{\omega}_{e}$ of the encounter frequency shows larger variations as a result of the broader frequency range the power spectral density $Z(\omega)$ spans over. Moreover the presence of multiple peaks of almost equal magnitude in the PSDs, as for $Z_{2}(\omega)$ and $Z_{3}(\omega)$, increases the difficulty of identifying the main frequency carrier.

Figure $7(\mathrm{c})$ shows that the estimator slightly overestimates the peak frequency of $Z_{1}(\omega)$, which can be explained by the skewness of the PSD towards the higher frequencies. Between 6 and 12 minutes the estimates varies between 0.5 and $0.7 \mathrm{rad} / \mathrm{s}$, which can be expected since $Z_{2}(\omega)$ shows not clear dominant peak during this time interval. For the last 5 minutes $\hat{\omega}_{e}$ first oscil- 

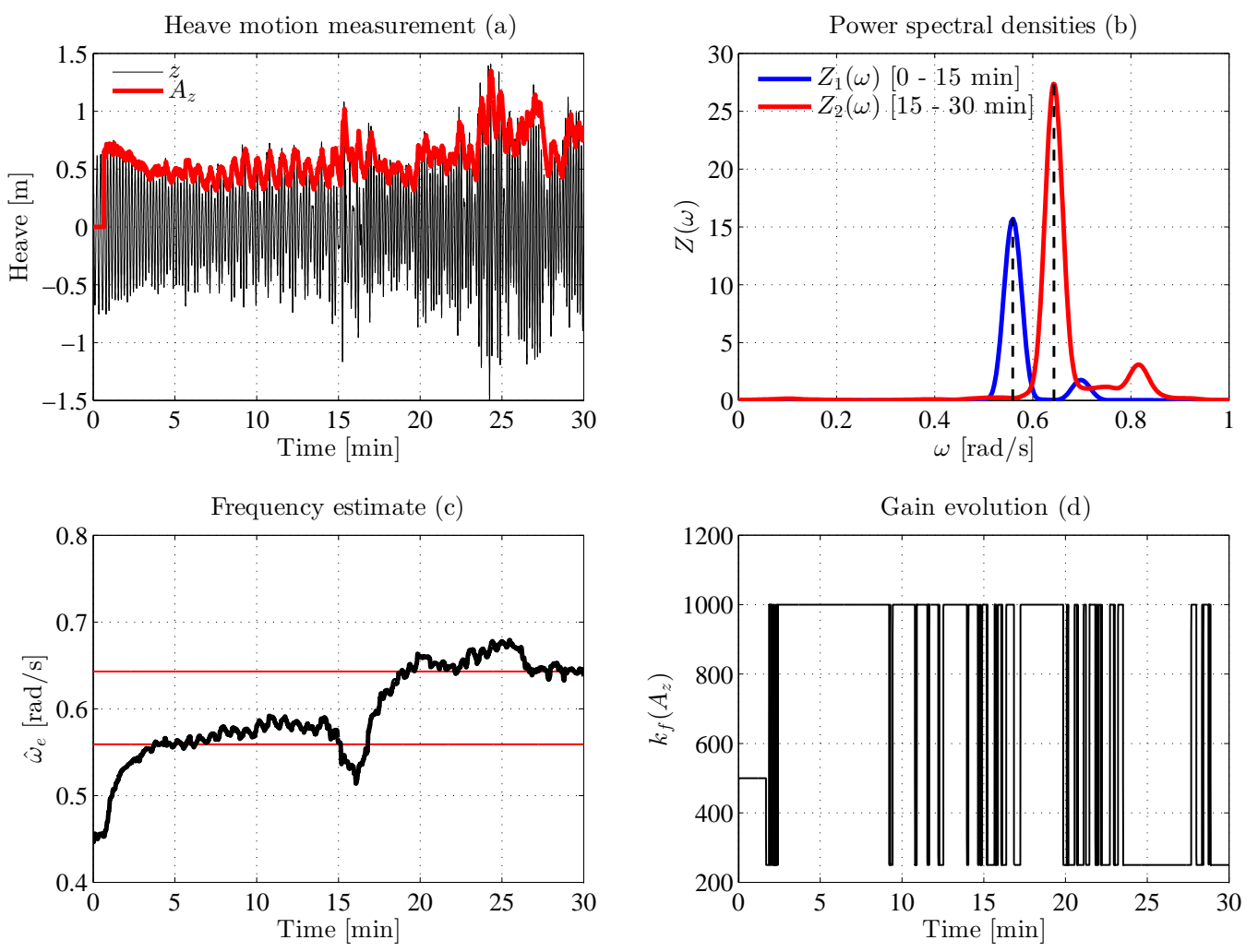

Figure 6: Test II: estimation of wave encounter frequency from heave displacement measurements collected during regular wave experiments $R-1173$ and $R-1189$. The encounter frequency estimator converges towards the true value of the two main peak frequencies of $Z(\omega)$, however the presence of side lobs in the power spectral density influences the estimation process pulling $\hat{\omega}_{e}$ towards slightly higher values.

lates around $0.6 \mathrm{rad} / \mathrm{s}$, and than decreases to around $0.5 \mathrm{rad} / \mathrm{s}$, which once again nearly matches the location of the two peaks of the power spectral density $Z_{3}(\omega)$.

In order to reduce these fluctuations a low-pass filter is added at the output of the switching-gain estimator. This smooths the behavior of the frequency estimate of the encounter wave, which now stays much closer to the true value at all times, as shown by the green line in Figure 7(c).

\subsection{Atlantic passage full-scale data}

The final test of the switching-gain frequency estimator is run on a data set of full-scale ship motions' data recorded on board the container ship 

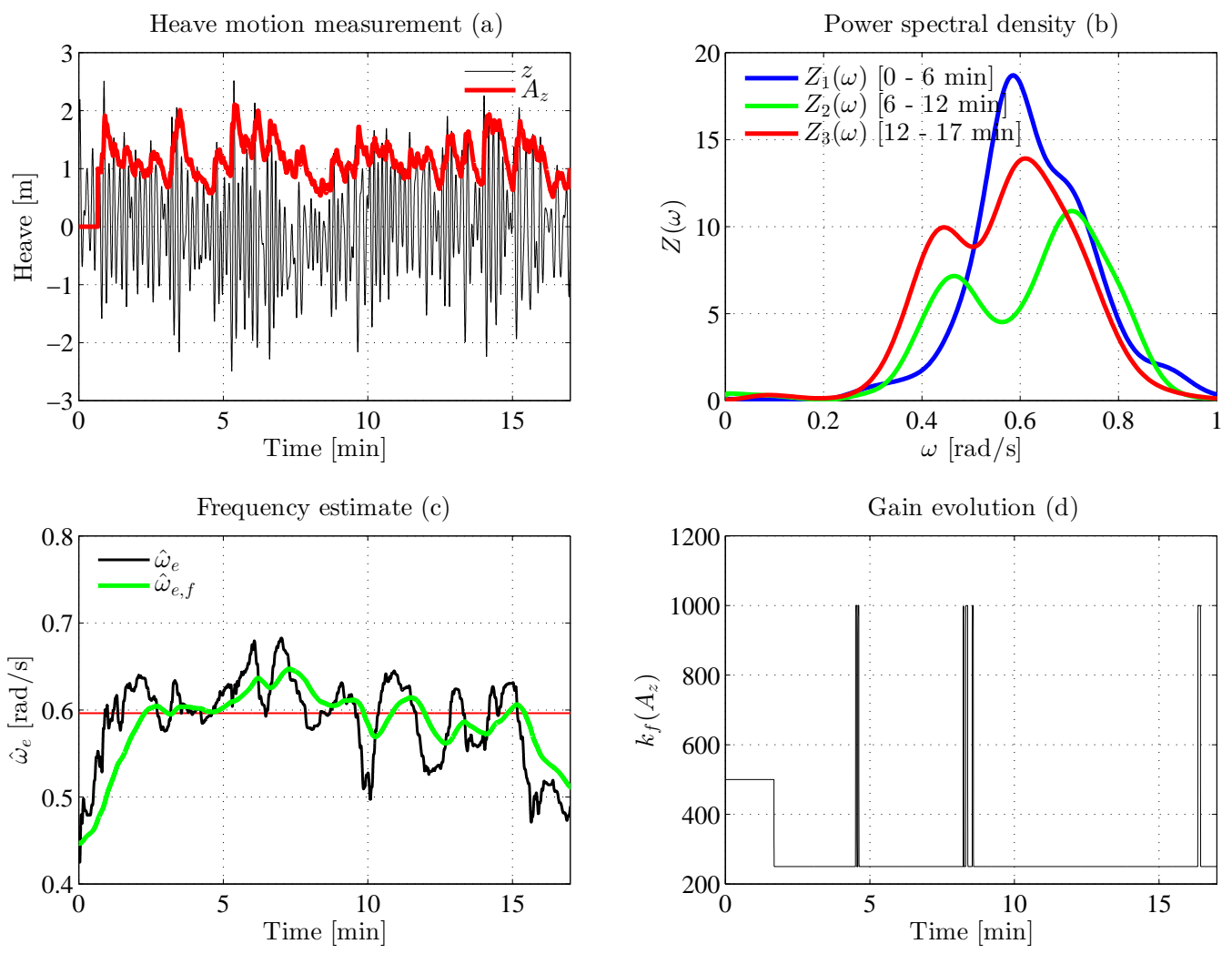

Figure 7: Test III: estimation of wave encounter frequency from heave displacement measurements collected during irregular wave experiment $I-1195$. The encounter frequency estimator converges towards the true value of the two main peak frequencies of $Z(\omega)$, however the presence of side lobs in the power spectral density influences the estimation process pulling $\hat{\omega}_{e}$ towards slightly higher values.

Clara Maersk crossing the North Atlantic Ocean during a storm. The length of the vessel was $197 \mathrm{~m}$ and its displacement volume was $33000 \mathrm{~m}^{3}$. Time series used here correspond to the pitch angle recorded during nine hours of navigation. Due to the larger amplitudes induced by the stormy weather different settings of the gain switching mechanism have been chosen. The settings for the filter and the gain switching mechanism are given in Table 4.

\subsubsection{Performance assessment on full-scale data}

The measurement of the pitch angle aboard the ship is presented in Figure 8(a). The measurements show that the amplitude of the pitch angle is fairly constant over a long period of time, with the exception of few larger 

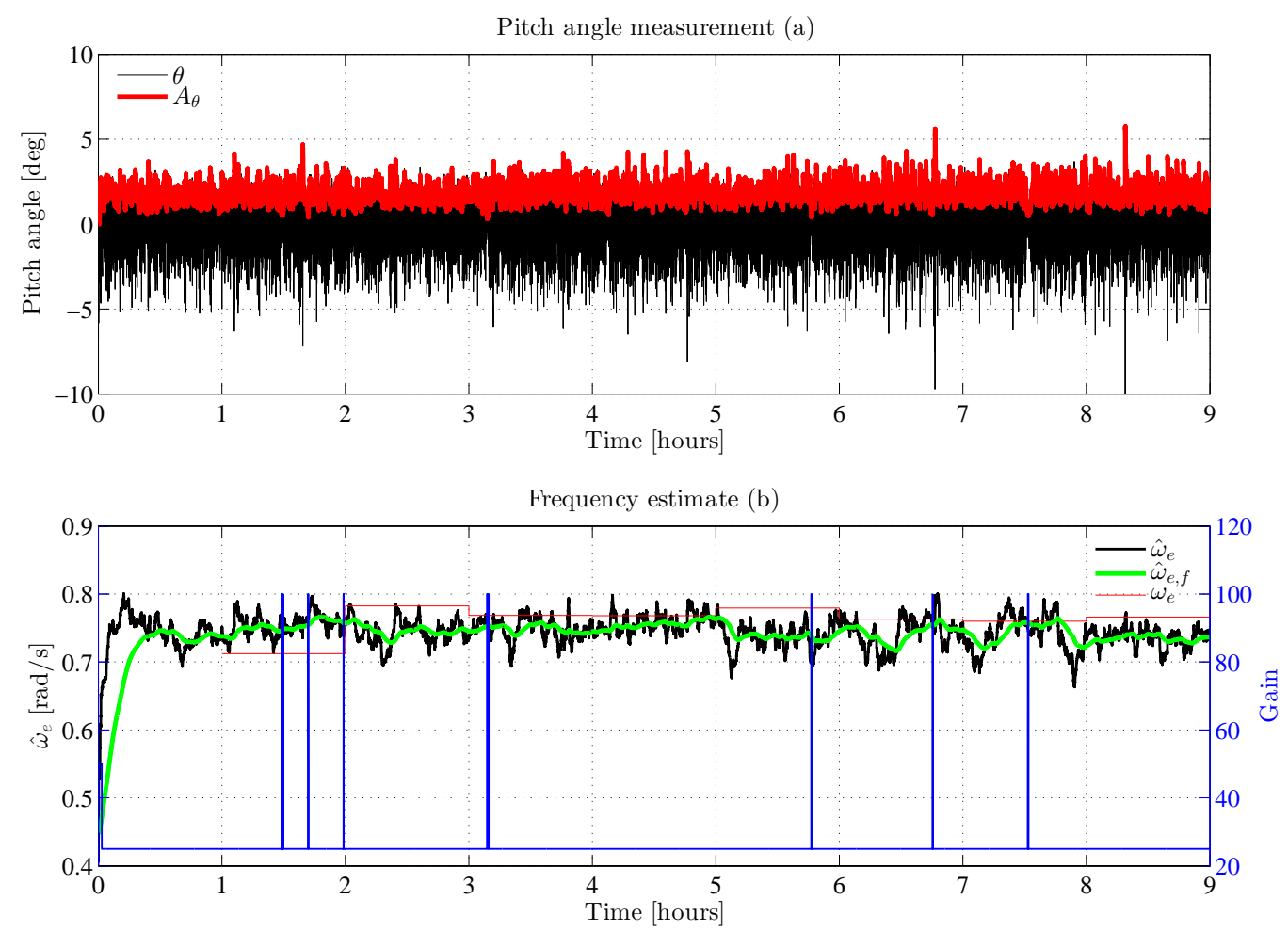

Figure 8: Test IV: estimation of wave encounter frequency from pitch angle measurements collected aboard Clara Maersk during a passage through the North Atlantic. The wave encounter frequency estimator succeeds in rapidly identifying the range of frequencies where the dominant spectral components fall into.

peaks. Figure 9 presents the evolution of the pitch power spectral density $\Theta(\omega)$ over the nine hours, and the wave encounter frequency identified as the frequency of the largest peak of each PSD. The power spectral density spans over a very broad range of frequencies with a multitude of peaks; however, the main spectral components are in the range $0.7-0.8 \mathrm{rad} / \mathrm{s}$.

The estimate of the encounter frequency based on the pitch measurement is given in Figure $8(\mathrm{~b})$, which shows that $\hat{\omega}_{e}$ is also in the range $0.7-0.8$ $\mathrm{rad} / \mathrm{s}$. Comparing the estimate of the encounter frequency with $\omega_{e}$ identified through the analysis of the power spectral density it can be noted that $\hat{\omega}_{e}$ converges to values in close proximity to $\omega_{e}$. Application of a low-pass filter to the output of the switching-gain frequency estimator helps in smoothing the obtained frequency estimate. Figure 8(b) also shows the switching gain 

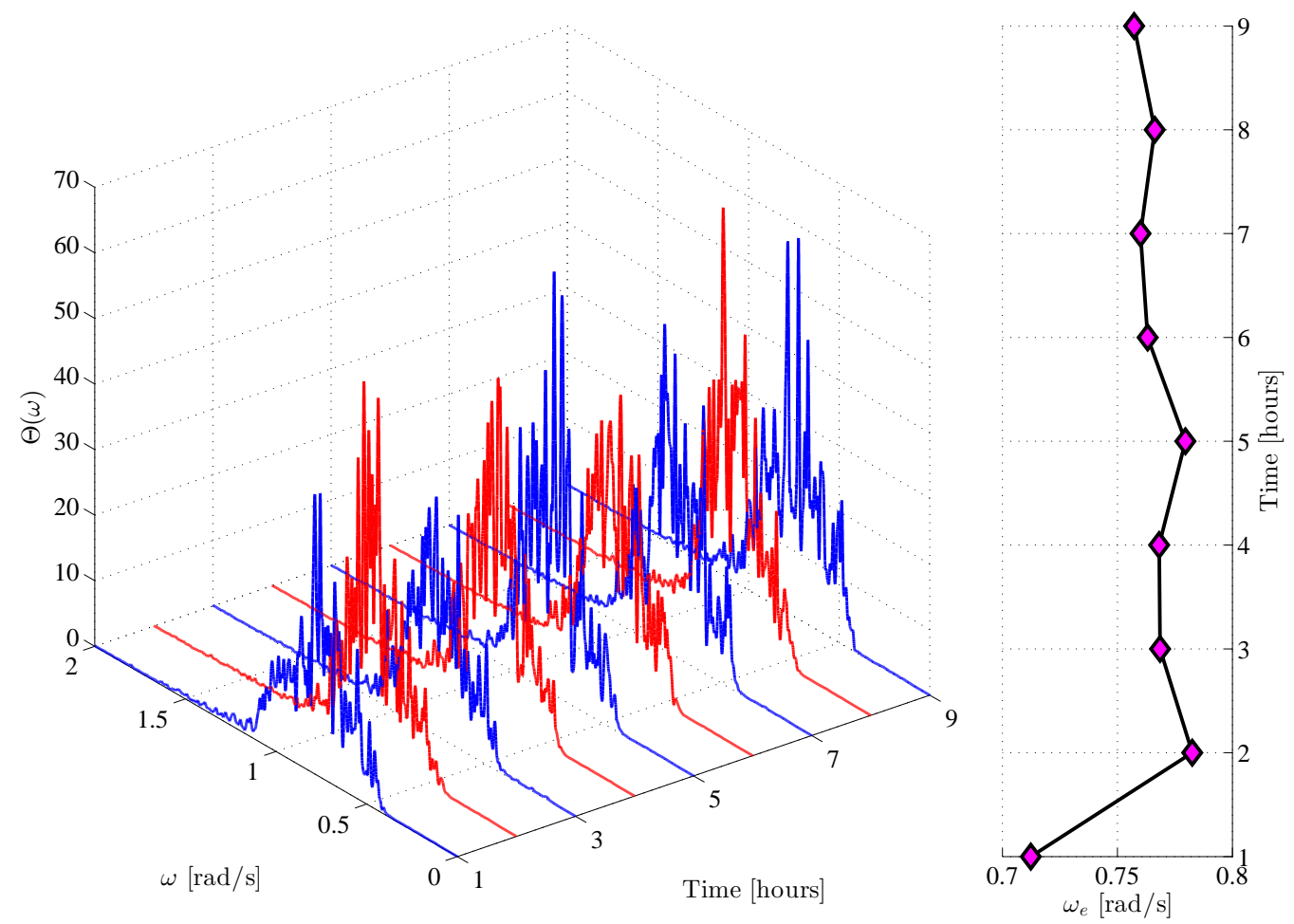

Figure 9: Evolution of the pitch power spectral density over 9 hours of navigation across a storm.

strategy. Since the amplitude $A_{\theta}$ of the measured pitch angle is rather constant and sufficiently exciting for rapid adaptation there are only few gain switches.

\section{Conclusions}

Knowledge of the parameters characterizing the sea state is of extreme value in order to carry out marine operations in a safe, reliable and cost effective ways. This paper has derived a signal-based nonlinear observer for the estimation of the wave encounter frequency. The kernel of the designed estimator is a second-order nonlinear observer with a switching-gain mechanism designed to estimate the frequency of a sinusoid with unknown frequency, amplitude and phase. The origin of the estimation error dynamics is proven to be global exponentially stable, extending previous results that only guaranteed global $\mathcal{K}$-exponential stability. 
Table 4: Switching-gain estimator parameter settings for full-scale data.

\begin{tabular}{lccc}
\hline Quantity & Symbol & Value & Unit \\
\hline Switching time constant & $T_{f}$ & 0.05 & $\mathrm{~s}$ \\
Filter cut-off frequency & $\omega_{f}$ & 1 & $\mathrm{rad} / \mathrm{s}$ \\
Switching amplitude pitch & $A_{\theta, 0}$ & 0.01 & $\mathrm{rad}$ \\
Initialization time & $t_{\text {init }}$ & 100 & $\mathrm{~s}$ \\
Initialization gain pitch angle & $k_{\text {init }}$ & 50 & - \\
Filter low gain pitch angle & $k_{\min }$ & 25 & - \\
Filter high gain pitch angle & $k_{\max }$ & 100 & - \\
\hline
\end{tabular}

The frequency estimator has been extensively tested on model-scale motion data of a container ship gathered during towing tank experiments in regular and irregular waves, and on full-scale motion data of a container ship recorded in an Atlantic passage during a storm. In all scenarios the nonlinear switching-gain frequency estimator succeeds in identifying the frequency range where the encounter frequency falls into. Extremely good results in terms of fast convergence and tracking are obtained for the model-scale data collected in the regular wave experiments, since the ship responses in pitch and heave closely resemble pure sinusoidal signals. The broadening of the spectral content of the heave and pitch responses recorded in the irregular wave experiment and in the sea trial clearly challenges the capabilities of the observer to converge to the true value of the wave encounter frequency. Nevertheless, the frequency estimator achieves its objective, by providing an estimate within the frequency range where the main spectral components are.

\section{Acknowledgment}

This work was partly supported by the Norwegian Research Council (project no. 223254) through the Center of Autonomous Marine Operations and Systems (AMOS) at the Norwegian University of Science and Technology. Data from model tests were kindly provided by Dr I. Drummen (Marin), Professor T. Perez (Queensland University of Technology) and Dr G. Storhaug (DNV-GL). Data from the Atlantic passage were provided by Professor M. Blanke (Technical University of Denmark) and they were presented with the permission from Maersk Lines. 


\section{References}

S. V. Aranovskiy, A. A. Bobtsov, A. S. Kremlev and G. V. Luk'yanova. A robust algorithm for identification of the frequency of a sinusoidal signal. J. of Computer and Systems Sciences Intern, Vol. 46, No. 3, 371-376, 2007.

S. V. Aranovskiy and A. A. Bobtsov. Output harmonic disturbance compensation for nonlinear plants. In Proceeding of the 20th Mediterranean Conference on Control and Automation, 386-391, 2012, Barcelona, Spain.

D. J. W. Belleter, D. A. Breu, T. I. Fossen and H. Nijmeijer. Nonlinear observer design for parametric roll resonance. In Proceeding of the 11th international conference on the stability of ships and ocean vehicles, 699705, 2012, Athens, Greece.

D. J. W. Belleter, D. A. Breu, T. I. Fossen and H. Nijmeijer. A globally $\mathcal{K}-$ exponentially stable nonlinear observer for wave encounter frequency. In: Proc. of the IFAC CAMS'2013, Osaka, Japan, 2013.

A. Bobtsov. New approach to the problem of globally convergent frequency estimator. International Journal of Adaptive Control Signal Processing, Vol. 22, 306-317, 2008.

M. Bodson and S. C. Douglas. Adaptive algorithms for the rejection of sinusoidal disturbances with unknown frequency. Automatica, Vol. 33, No. 12, 2213-2221, 1997.

H. Enshaei and R. Brimingham. Monitoring Dynamic Stability via Ship's Motion Responses. In Proceeding of the 11th international conference on the stability of ships and ocean vehicles, 707-717, 2012, Athens, Greece.

T. I. Fossen. Handbook of Marine Craft Hydrodynamics and Motion Control. Wiley, 2011.

T. I. Fossen and J. P. Strand. Passive nonlinear observer design for ships using Lyapunov methods: full-scale experiments with a supply vessel. $A u$ tomatica, Vol. 35, No. 1, 3-16, 1999.

V. Hassani, A. M. Pascoal and A.J. Sørensen. A novel methodology for adaptive wave filtering: theory and experiments. In Proceeding of the 52nd IEEE conference on decision and control, 2162-2167, 2013, Florence, Italy. 
C. Holden, R. Galeazzi, C. Rodríguez, T. Perez, T. I. Fossen, M. Blanke and M. A. S. Neves. Nonlinear Container Ship Model for the Study of Parametric Roll Resonance. Modeling, Identification and Control, Vol. 28, No. 1, 87-113, 2007.

M. Hou. Parameter identification of sinusoids. IEEE Transactions of Automatic Control, Vol. 57, No. 2, 467-472, 2012.

L. Hsu, R. Ortega and G. Damm. A globally convergent frequency estimator. IEEE Transactions on Automatic Control, Vol. 44, No. 4, 698-713, 1999.

K. H. Khalil. Nonlinear Systems.Prentice-Hall, 2002.

A. Loria and E. Panteley (2004). Cascaded nonlinear time-varying systems: analysis and design. Ch. 2. In: Advanced Topics in Control Systems Theory, Springer-Verlag London (F. Lamnabhi-Lagarrigue, A. Loria and E. Panteley Eds.), pp. 23-64, 2004.

R. Marino and P. Tomei. Global estimation of $\mathrm{n}$ unknown frequencies. IEEE Transactions on Automatic Control, Vol. 47, No. 8, 1324-1328, 2002.

U. D. Nielsen. Estimations of on-site directional wave spectra from measured ship responses. Marine Structures, Vol. 19. No. 1, 33-69, 2006.

V. Nikiforov. Adaptive servomechanism controller with an implicit model. International Journal of Control, Vol. 68, 277-286, 1997.

P. Regalia. An Improved Lattice-based Adaptive IIR Notch Filter. IEEE Trans. on Signal Processing, Vol. 39, 2124-2128, 1991.

A. Stotsky. Frequency determination in control applications: excitationbased approach. Journal of Systems and Control Engineering, Vol. 226, No. 8, 1142-1148, 2012.

O. J. Sørdalen and O. Egeland. Exponential stabilization of nonholonomic chained systems. IEEE Transactions on Automatic Control, Vol. 40, No. $1,1995$.

E. A. Tannuri, J. V. Sparano, A. N. Simos and J. J. Da Cruz. Estimating directional wave spectrum based on stationary ship motion measurements. Applied Ocean Research, Vol. 25, 246-261, 2003. 
T. Iseki and K. Ohtsu. Bayesian estimation of directional wave spectra based on ships motions. Control Engineering Practice, Vol. 8, 215-219, 2000.

X. Xia. Global frequency estimation using adaptive identifiers. IEEE Transactions on Automatic Control, Vol. 47, No. 7, 1188-1193, 2002. 